\title{
Two-dimensional dilaton gravity and spacetimes with finite curvature at the horizon away from the Hawking temperature
}

\author{
O. B. Zaslavskii \\ Department of Physics, Kharkov State University, Svobody Sq.4, Kharkov \\ 31007r, Ukraine \\ E-mail: aptm@kharkov.ua
}

\begin{abstract}
It is shown that static solutions with a finite curvature at the horizon may exist in dilaton gravity at temperatures $T \neq T_{H}$ (including $T=0$ ) where $T_{H}$ is the Hawking one. Hawking radiation is absent and the state of a system represents thermal excitation over the Boulware vacuum. The horizon remains unattainable for a observer because of thermal divergences in the stress-energy of quantum fields there. However, the curvature at the horizon is finite, when measured from outside, since these divergences are compensated by those in gradients of a dilaton field. Spacetimes under consideration are geodesically incomplete and the coupling between dilaton and gravity diverges at the horizon, so we have "singularity without singularity".
\end{abstract}

PACS: 04.60Kz, 04.70.Dy

In recent years a great interest focused on two-dimensional (2D) theories of dilaton gravity. One of the reason of this consists in the possibility to trace in detail the process of black hole evaporation since on the semiclassical level spacetime evolution is described by differential equations directly following from the Lagrangian even if quantum effects are taken into account [1]. It turned out that such a kind of theories possesses a rather rich set of exactly solvable models. These circumstances give us hope to get insight into subtle features of black hole evolution. Especially important is the question whether final geometry can be regular or not. In Ref. [2] (BPP) the exactly solvable model was proposed in which, under certain choice of parameters, a black hole leaves after evaporation everywhere regular geodesically complete geometry which infinitely extends to a region of strong coupling ("semi-infinite" 
throat). In so doing, quantum fields are in the radiationless state in which effects of vacuum polarization tend to zero at Minkowski infinity. In Ref. [3] (CN) more general model was suggested interpolating between the BPP and RST [4] ones. The qualitatively new feature of the $\mathrm{CN}$ model consists in that, although a static radiationless geometry with curvature finite everywhere is still possible, it becomes geodesically incomplete.

Meanwhile, the CN model possesses some other intriguing features which were not noticed in [3]. Namely, we will show below that at the boundary of spacetime $g_{00} \rightarrow 0$, so it represents a Killing horizon. At this point stresses of quantum fields due to back reaction diverge since the solution under discussion is radiationless (temperature $T=0$ ) whereas the usual condition of their finiteness demands $T=T_{H}$ where $T_{H}$ is the Hawking temperature (see, for instance, [5]). Thus, the intimate connection between regularity of a geometry on a horizon and equality of temperatures $T=T_{H}$ ceases to exist and we have a static geometry with a finite curvature at the horizon at $T \neq T_{H}$ and the infinite quantum stresses! In this respect the significance of the $\mathrm{CN}$ model is beyond the context of concrete problems of $2 \mathrm{D}$ dilaton gravity: relative simplicity of the $2 \mathrm{D}$ case enables us in the best possible way to review unbiasedly some fundamentals of black hole physics and elucidate opportunities which probably may occur in a $4 \mathrm{D}$ world but remain hidden because of complexity of the situation in the latter case.

In the present paper we argue also that there exists a whole range of temperatures for which properties of spacetime sketched above hold true. We exploit exactly solvable models of 2D gravity [6], [7] (with respect to which the $\mathrm{CN}$ one is the particular case) and show that the results remain valid for a whole class of such theories provided the coupling between gravitation and dilaton obeys some general restrictions.

Let us consider the system described by the action

$$
I=I_{0}+I_{P L}
$$

where

$$
I_{0}=\frac{1}{2 \pi} \int_{M} d^{2} x \sqrt{-g}\left[F(\phi) R+V(\phi)(\nabla \phi)^{2}+U(\phi)\right]
$$


$I_{P L}$ is the Polyakov-Liouville action [8] incorporating effects of Hawking radiation and its back reaction on the background metric for a multiplet of $\mathrm{N}$ scalar fields, and the boundary terms omitted. In what follows we will use the quantities $T_{\mu \nu}$ defined according to

$$
\delta I=\frac{1}{2} \int d^{2} x \sqrt{|g|} \delta g^{\mu \nu} T_{\mu \nu}
$$

so the filed equations which are obtained by varying a metric have the form $T_{\mu \nu}=0$.

In the conformal gauge

$$
d s^{2}=-e^{2 \rho} d x_{+} d x_{-}
$$

the Polyakov-Liouville action reads $I_{P L}=-\frac{2 \kappa}{\pi} \int d^{2} x \partial_{+} \rho \partial_{-} \rho=\frac{\kappa}{\pi} \int d^{2} x \sqrt{g}(\nabla \rho)^{2}$. After integration by parts the action (四) is reduced (up to boundary terms) to $I=$ $\frac{1}{2 \pi} \int d^{2} x \sqrt{g}\left[V(\nabla \phi)^{2}+2 \nabla \rho \nabla F+2 \kappa(\nabla \rho)^{2}+4 \lambda^{2} e^{\eta}\right]$, where by definition $\eta=\int d \phi \omega, \omega=U^{\prime} / U$. In what follows we restrict ourselves by exactly solvable models of dilaton gravity [6], [7] that implies the constraint on the form of the potential $V=\omega\left(u-\frac{\kappa \omega}{2}\right)+\gamma(u-\kappa \omega)^{2}$ where $u=F^{\prime}$. In what follows we consider only the simplest choice $\gamma=0$, so

$$
V=\omega\left(u-\frac{\kappa \omega}{2}\right)
$$

Introducing new fields $\Omega$ and $\chi$ instead of $\phi$ and $\rho$ according to $\tilde{F} \equiv F-\kappa \eta=2 \kappa \Omega$, $\eta=2(\chi-\Omega-\rho)$, after simple rearrangement we obtain $I=\frac{1}{\pi} \int d^{2} x \sqrt{g}\left\{\kappa\left[(\nabla \chi)^{2}-(\nabla \Omega)^{2}+\right.\right.$ $\left.2 \lambda^{2} e^{2(\chi-\Omega-\rho)}\right\}$. Corresponding equations of motion have the form $2 \kappa \partial_{+} \partial_{-} \Omega=-\lambda^{2} e^{2(\chi-\Omega)}$, $2 \kappa \partial_{+} \partial_{-} \chi=-\lambda^{2} e^{2(\chi-\Omega)}$. One can choose the gauge $\chi=\Omega$ whence $\partial_{+} \partial_{-} \tilde{F}=-\lambda^{2}$. This equation should be supplemented by the constraint equations $T_{++}=T_{--}=0\left(T_{\mu \nu}=2 \frac{\delta I}{\delta g^{\mu \nu}}\right)$. The expressions for the classical parts of $T_{++}$and $T_{--}$follow directly from the general expression for covariant components

$$
T_{\mu \nu}^{(0)}=\frac{1}{2 \pi}\left\{2\left(g_{\mu \nu} \square F-\nabla_{\mu} \nabla_{\nu} F\right)-U g_{\mu \nu}+2 V \nabla_{\mu} \phi \nabla_{\nu} \phi-g_{\mu \nu} V(\nabla \phi)^{2}\right\},
$$

which can be obtained by varying the action $I_{0}$. The formula for the quantum contribution can be obtained from the conservation law and conformal anomaly and has the form 


$$
T_{ \pm \pm}^{(P L)}=\frac{2 \kappa}{\pi}\left[\left(\partial_{ \pm} \rho\right)^{2}-\partial_{ \pm}^{2} \rho+t_{ \pm}\right]
$$

where the function $t_{ \pm}\left(x_{ \pm}\right)$are determined by the boundary conditions. From (16) and (7) we have the equation $\partial_{ \pm}^{2} \tilde{F}=2 \kappa t_{ \pm}$. To find the explicit form of $t_{ \pm}$, let us impose the condition that at the right infinity quantum fields should be at a finite temperature $T$. Then in asymptotically flat coordinates $\sigma_{ \pm}=t \pm \sigma$ connected with $x_{ \pm}$according to $\lambda x_{ \pm}= \pm e^{ \pm \lambda x_{ \pm}}$ stresses take the form $T_{ \pm \pm}^{(\sigma)}=-\pi T^{2} / 12$ at $\sigma \rightarrow \infty$ where $d s^{2}=-d \sigma_{+} d \sigma_{-}$. On the other hand, asymptotically $2 \rho \approx-\ln \left(-\lambda^{2} x_{+} x_{-}\right)$to match the region of a linear dilaton vacuum $\phi=-\sigma \lambda$. Substituting this into (7) and performing transformation of tensor components between two coordinate systems, we have $t_{ \pm}=\frac{1}{4} x_{ \pm}^{-2}\left(1-\frac{T^{2}}{T_{0}^{2}}\right)$. Then, integrating the equations of motion for $\tilde{F}$ we obtain

$$
\begin{gathered}
d s^{2}=g\left(-d t^{2}+d \sigma^{2}\right), g=\exp (2 \rho+2 y), y=\lambda \sigma, 2 \rho=-\int \omega d \phi . \\
\tilde{F}(\phi)=f(y) \equiv e^{2 y}-B y+C, B=\kappa\left(1-T^{2} / T_{0}^{2}\right), T_{0}=\lambda / 2 \pi .
\end{gathered}
$$

The expressions (8) describe generic static configurations gravitational and dilaton fields in exactly solvable models of dilaton gravity at finite temperatures. Hereafter we concentrate ourselves on $\tilde{F}(\phi)$ such that $\tilde{F}(\phi)$ has one simple minimum at some real $\phi_{0}$ where $\tilde{F}^{\prime}\left(\phi_{0}\right)=0$. The well known example is the RST model [4]. Let the coefficient $B>0$. The structure of spacetime depends crucially on the relationship between $f_{\min }=f\left(y_{0}\right)$ (the minimum value of $f(y)$ achieved in the point $\left.y_{0}\right)$ and $\tilde{F}\left(\phi_{0}\right)$. It follows from (8) that $\frac{d \phi}{d y}=\frac{f^{\prime}(y)}{\tilde{F}^{\prime}(\phi)}$. If $f_{\min }<$ $\tilde{F}\left(\phi_{0}\right)$, the dilaton value changes monotonically from $\phi=-\infty$ at right infinity $y=\infty$ to $\phi=\phi_{0}$ where the spacetime is singular and cannot be continued further. For the RST model the structure of spacetime at the equilibrium temperature $T=T_{0}$ is analyzed in detail in [9] (see also generalization in [7]). If $f_{\min }>\tilde{F}\left(\phi_{0}\right)$, the dilaton field takes its values in the limits $\left(-\infty, \phi_{1}\right)$ where $\phi_{1}<\phi_{0}, \tilde{F}^{\prime}(\phi)$ changes its sign nowhere.

In what follows we dwell upon on the special case $\tilde{F}\left(\phi_{0}\right)=f_{\min }$, so $C=C^{*}$ where

$$
C^{*}=\tilde{F}\left(\phi_{0}\right)+C_{0}, C_{0}=-\frac{B}{2}\left(1+\ln \frac{2}{B}\right)
$$


Then $f^{\prime}(y)$ and $\tilde{F}^{\prime}(\phi)$ turn into zero simultaneously and $\frac{d \phi}{d y}$ does not changes its sign, so the dependence $\phi(y)$ is monotonic. As a result, $\phi \rightarrow \infty$ when $y \rightarrow-\infty$, i.e. at the event horizon. In the vicinity of the point $y_{0}$ one can use the power expansion, so the equality $\tilde{F}(\phi)=f(y)$ turns into $\frac{\tilde{F}^{\prime \prime}\left(\phi_{0}\right)}{2}\left(\phi-\phi_{0}\right)^{2}+\frac{\tilde{F}^{\prime \prime \prime}\left(\phi_{0}\right)^{3}}{6}\left(\phi-\phi_{0}\right)^{3}+\ldots=\frac{f^{\prime \prime}\left(y_{0}\right)}{2}\left(y-y_{0}\right)^{2}+\frac{f^{\prime \prime \prime}\left(y_{0}\right)}{6}\left(y-y_{0}\right)^{3}+\ldots$ whence it is clear that the Riemann curvature $R=-\lambda^{2} g^{-1} \frac{d}{d y}\left(g^{-1} \frac{d g}{d y}\right)=-2 \lambda^{2} \frac{d^{2} \phi}{d y^{2}} e^{-2 \phi-2 y}$ is finite in the point $y_{0}$.

Let us consider the concrete example. We assume that the functions $f(y)$ and $\tilde{F}(\phi)$ obey the conditions described above which guarantee the absence of the singularity outside the horizon. We do not specify the exact form of $\tilde{F}(\phi)$ in the whole region and only assume that in the vicinity of the horizon it reads

$$
\tilde{F} \approx e^{-2 \phi}+b \phi, \phi \rightarrow \infty
$$

The condition (5) of exact solvability implies that $V \approx\left(b-2 e^{-2 \phi}\right) \omega+\frac{\kappa \omega^{2}}{2}$ near the horizon. We find that at $y \rightarrow-\infty$

$$
\phi \rightarrow \infty, g \sim e^{2|y|(B / b-1)}, R \sim e^{-2|y|(2 B / b-1)}
$$

Thus, if $B / b<1$ the surface $y=-\infty$ represent a horizon where $g=0$. In the Schwarzschild gauge $x=\int d \sigma g, d s^{2}=-g d t^{2}+g^{-1} d x^{2}$ we have an usual behavior $g \sim x-x_{h}$ near the horizon located at $x=x_{h}$. If $2 B / b \geq 1$, the Riemann curvature on this surface is finite. It is remarkable that both conditions are consistent with each other. Thus, if $\frac{1}{2} \leq \frac{B}{b}<1$ or, equivalently,

$$
1-\frac{b}{\kappa}<\frac{T^{2}}{T_{0}^{2}} \leq 1-\frac{b}{2 \kappa}
$$

we have a black hole with the Riemann curvature finite everywhere including the horizon. This inequality is not satisfied by $T=T_{0}$ when, as is well known, a horizon is regular (the usual Hartle-Hawking state). However, there is no contradiction here since at $T=T_{0}$ the coefficient $B=0$ and asymptotic behavior of such a solution [7], [9] has nothing to do with (11). Thus, according to (12), the Hartle-Hawking state is not included in the set of states 
under discussion and there are two possibilities to have the horizon with a finite curvature which cannot match continuously: $T=T_{0}$ (the isolated point) or $T$ obeys the inequality (12) (the whole range of temperatures).

In the particular case of the RST model $b=\kappa$, so (12) is reduced, according to (8), to the condition

$$
0<T \leq \frac{T_{0}}{\sqrt{2}}
$$

The case $T=0$ is now trivial since for solutions under discussion $C=0$ according to (9), eq. (8) gives us $e^{-2 \phi}+\kappa \phi=e^{2 y}-\kappa y$ and, with the condition of asymptotical flatness $\phi(\infty)=-\infty$, we have a linear dilaton vacuum solution $\phi=-y$ with a flat spacetime. Therefore, for the RST model a horizon with a finite curvature may exist only at nonzero temperature. However, for a generic $\kappa<b \leq 2 \kappa$ it is possible for such a horizon to exist even at $T=0$.

The case $T=0$ is tractable for more detailed investigation. Let us consider the example

$$
\tilde{F}=e^{2 \gamma(\phi)}-\kappa \gamma(\phi)
$$

Then, taking into account that for this model the quantity $C^{*}$ depends on temperature according to $C^{*}=C_{0}(0)-C_{0}(T)$, so $C^{*}=0$ at $T=0$, we find from (8) the solution $y=\gamma(\phi)$. Let us choose, for instance,

$$
\gamma=-\phi-\frac{1}{2} \ln \left(1+e^{2 \phi}\right)
$$

Then $g=\left(1+e^{2 \phi}\right)^{-1}$. After simple calculations we find the Riemann curvature $R=$ $4(1-g)(g-2)^{-3}$. At the horizon $\phi=\infty, g=0$ the curvature remains finite in spite of quantum stresses diverge there. For instance, $T_{y}^{y(P L)}=-\frac{\lambda^{2}}{24}\left(1+\frac{1}{2} e^{-2 \phi}\right)^{-2} g^{-1}$.

Thus, we arrive at a rather surprising conclusion: spacetimes with a finite curvature at the horizon may exist at a temperature different from the Hawking one and, moreover, their temperature may be equal to zero! This conclusion is the main result of the present paper.

What is the physical reason for such an unusual behavior? It is instructive to look at fields equations $T_{\mu}^{(0) \nu}+T_{\mu}^{(P L) \nu}=0$. The classical part of the effective stress-energy 
tensor $T_{\mu}^{(0) \nu}$ contains terms with gradients of the dilaton field $\phi$. For instance, $T_{x}^{(0) x}=$ $(2 \pi)^{-1}\left[V(\nabla \phi)^{2}-U+2 \nabla^{0} \nabla_{0} F\right]$. A simple estimate based on (10), (11) shows that near the horizon where $y \rightarrow-\infty$ and $\phi \approx-\frac{B}{b} y, T_{x}^{(0 x 1} \sim g^{-1} \rightarrow \infty$. On the other hand, in the Scwharzschild coordinates we have $T_{x}^{(P L) x}=-\frac{\pi}{6}\left[T^{2}-\frac{1}{4 \pi}\left(\frac{d g}{d x}\right)^{2}\right] g^{-1} \sim g^{-1} \rightarrow \infty$ if $T \neq T_{H}$ [5]. Thus, each part of $T_{\mu}^{\nu}$ diverges separately and a regular metric near the horizon arises as a result of mutual compensation of these divergences. Meanwhile, the usual proof of the fact that $T=T_{H}$ relies strongly upon the regularity of the stress-energy tensor of quantum field at the horizon. The corresponding criteria [5] appeal to the behavior of $T_{\mu}^{(P L) \nu}$ but do not take into account properties of $T_{\mu}^{(0) \nu}$, i.e. they were considered in isolation from the dynamic contents of the theory whose solution a given metric represents. Usually, such an approach is justified since a classical part of field equation is regular, as was tacitly assumed in [5, but the present case is exceptional in that either quantum or classical contributions to field equations diverge near the horizon.

Thus, the curvature may be finite everywhere even when the stress-energy tensor describing the back reaction of quantum fields diverges on the horizon. The situation is sharply contrasted with that in general relativity where the stress-energy tensor singular at a horizon is inconsistent with the regularity of a metric and of the Einstein tensor. The reason consists in that now we have, apart from a metric, one more classical field - the dilaton one coupled to a metric. Gradients of this field compensate the divergencies due to back reaction of quantum fields.

It can be readily seen from the above formulas that the proper distance between the horizon and any other point $l=\lambda \int_{-\infty}^{y} d y \sqrt{g}$ is finite. Therefore, the spacetime region outside the horizon is geodesically incomplete. The dilaton field $\phi$ cannot be continued further across the horizon since $\phi$ diverges there. The situation with continuation across such a surface leads, in general, to complex dilaton values [10]. To avoid complex dilaton field, one may try to redefine the dilaton field choosing, say, $x(\phi)$ instead of $\phi$ where $x$ is a Schwarzschild-like coordinate. However, any redefinition cannot abolish the fact that 
the quantity $\tilde{F}$ describing the coupling between the dilaton and curvature diverges at the horizon. There is more deep reason to believe that any attempts of continuation across the horizon for our solutions should be rejected. The point is that any observer approaching the horizon from outside moves along a non-geodesic path and perceives fluxes of quantum field which become infinite at the horizon. Therefore, the horizon remains in fact unaccessible. The surface $\phi=\infty$ acts as a boundary of spacetime and the usual criteria of geodesic completeness is physically irrelevant for the solution under discussion. Of course, nothing prevents making a formal analytic continuation of the metric itself but, if the dynamic contents of the theory (interaction between gravitational, dilaton and quantum scalar fields) is taken into account, the region inside a horizon seems not to have a physical meaning for the model in question - at least, if one starts from the outside region. Thus, actually the loss of information inside the horizon is much more severe than in an usual case where at least an observer brave enough to dive into a black hole can get information about the region inside the horizon. In fact the notion of an event horizon changes its ordinary meaning since there are no events inside a horizon at all.

It is seen from (10) that $\tilde{F}$ diverges at the horizon where $\phi=\infty$ and so does the total entropy including the contribution of quantum fields which for exactly solvable models is proportional (up to a constant) to the horizon value of this function [7]. These divergences have the same nature as those in the Schwarzschild background where entropy of quantum fields is infinite if $T \neq T_{H}$. The difference between these two situations manifests itself, however, in that for a black-hole metric in general relativity disparity between two temperatures makes a horizon singular and in fact destroys it completely whereas in our case the horizon remains regular in the sense that the Riemann curvature remains finite there.

The features of spacetime discussed above are shared by a whole classes of exactly solvable models of dilaton gravity for which (i) $\tilde{F}^{\prime}(\phi)$ has a simple zero at some $\phi_{0}$, (ii) $\tilde{F} \sim \phi$ at $\phi \rightarrow \infty$, (iii) the constant in the solution is chosen $C=C^{*}$ in accordance with (9). These features, however, have nothing to do, for example, with the BPP model for which $\tilde{F}=e^{-2 \phi}$ 
and the choice $C=C^{*}$ leads to a regular semi-infinite "throat" [2] (generalization to a finite temperature is considered in [11]). The fact that unusual properties of the solution under discussion are intimately connected with quantum terms in the action reveals itself in the eq. (8) and its classical limit directly. Indeed, it follows from (8), (10) that in the limit $\kappa=0$ the point $\phi_{0}$ where $\tilde{F}^{\prime}=0$ moves to $\phi_{0}=\infty$, the coefficients $B=0=C^{*}, b \sim \kappa=0$ and eq. (8) with $C=C^{*}$ turns into $\tilde{F}=e^{-2 \phi}=e^{2 y}$ whose solution is a linear dilaton vacuum $\phi=-y$.

Usually, the temperature of Hawking radiation in a black hole background is determined by characteristics of a spacetime (say, an event horizon radius of a Schwarzschild hole). The fact that temperature may be arbitrary actually means that Hawking radiation as such is absent, so we deal with thermal excitation over the Boulware state which can be treated at any temperature. In particular, the choice $T=0$ corresponds to the Boulware vacuum. Therefore, the type of solution considered in the present paper might shed light to the fate of a black hole after evaporation, being a candidate on the role of a "remnant". This, however, needs further treatment based on a dynamic scenarios and is beyond the scope of the present paper.

While usually the notion of singularity implies that it is geometry which exhibits singular behavior, in our case divergencies manifest themselves in the dynamic characteristics (components of the stress-energy tensor) and in the coupling between the dilaton filed and curvature. These divergences along with the geodesic incompleteness of the metric in fact mean that the solution in question is singular in spite of the metric itself is regular - at least, in the restricted sense: as one approaches the horizon from outside, the Riemann curvature remains finite. So, we have "singularity without singularity".

In fact, we are faced with a qualitatively new type of objects in gravitation which occupies an intermediate place between regular black holes and naked singularities. It is of interest to elucidate whether coupling between gravitation and dilaton or other classical fields can produce the same type of solutions in the four-dimensional case.

I am grateful to Sergey Solodukhin for fruitful discussion and to Alessandro Fabbri for 
helpful correspondence.

[1] C. G. Callan, S. Giddings, J. A. Harvey, and A. Strominger, Phys. Rev. D 45 , R1005 (1992); T.Banks, A. Dabholkar, M. R. Douglas, and M. O. 'Loughlin, Phys. Rev. D 45, 3607 (1992).

[2] S. Bose, L. Parker, and Y. Peleg, Phys. Rev. D 52 (1995) 3512.

[3] J. Cruz and J. Navarro-Salas, Phys. Lett. B 375, 47 (1996).

[4] J. G. Russo, L. Susskind, and L. Thorlacius, Phys. Rev. D 46 (1992) 3444 ; Phys. Rev. D 47 (1992) 533.

[5] D. J. Lorantz, W. A. Hiscock, and P. R. Anderson, Phys.Rev. D 52, 4554 (1995).

[6] Y. Kazama, Y. Satoh, and A. Tsuichiya, Phys. Rev. D 51, 4265 (1995).

[7] O. B. Zaslavskii, Phys. Rev. D 59, 084013 (1999).

[8] A. M. Polyakov, Phys. Lett. B 103, 207 (1981).

[9] S. N. Solodukhin, Phys. Rev. D 53, 824 (1996).

[10] R. Balbinot and A. Fabbri, Class. Quant. Grav. 13, 2457 (1996).

[11] O. B. Zaslavskii, Phys. Lett. B 459 (1999) 105. 